\title{
Molecular-beam epitaxy of $\mathrm{CrSi}_{2}$ on $\mathrm{Si}(111)$
}

\author{
R.W. Fathauer, P.J. Grunthaner, and T. L. Lin \\ Jet Propulsion Laboratory, California Institute of Technology, Pasadena, California 91109 \\ K.T. Chang and J.H. Mazur \\ Materials Science Department, University of Southern California, Los Angeles, California 90089-0241 \\ D. N. Jamieson \\ Division of Applied Physics, California Institute of Technology, Pasadena, California 91125
}

(Received 9 September 1987; accepted 17 November 1987)

\begin{abstract}
Chromium disilicide layers have been grown on $\mathrm{Si}(111)$ in a commercial molecular-beam epitaxy machine. Thin layers $(10 \mathrm{~nm})$ exhibit two epitaxial relationships, which have been identified as $\mathrm{CrSi}_{2}(0001) / / \mathrm{Si}(111)$ with $\mathrm{CrSi}_{2}[10 \overline{10}] / / \mathrm{Si}[10 \overline{1}]$, and $\mathrm{CrSi}_{2}(0001) / / \mathrm{Si}(111)$ with $\mathrm{CrSi}_{2}[11 \overline{20} 0] / / \mathrm{Si}[10 \overline{1}]$. The latter case represents a $30^{\circ}$ rotation of the $\mathrm{CrSi}_{2}$ layer about the $\mathrm{Si}$ surface normal relative to the former case. Thick $(210 \mathrm{~nm})$ layers were grown by four different techniques, and the best-quality layer was obtained by codeposition of $\mathrm{Cr}$ and $\mathrm{Si}$ at an elevated temperature. These layers are not single crystal; the largest grains are observed in a layer grown at $825^{\circ} \mathrm{C}$ and are $1-2 \mu \mathrm{m}$ across.
\end{abstract}

\section{INTRODUCTION}

Heteroepitaxy of a variety of materials on Si has been demonstrated, including metals, insulators, and other semiconductors. In the case of semiconductors, the growth of $\mathrm{Ge},{ }^{1}$ SiGe alloys, ${ }^{2}$ and $\mathrm{GaAs}^{3}$ has been explored in detail. Both $\mathrm{Ge}$ and $\mathrm{GaAs}$ have lattice mismatches with $\mathrm{Si}$ of $\sim 4 \%$, and therefore are only suitable for devices localized near the surface of a thick epitaxial semiconductor layer. For devices requiring semiconductor heterojunctions, SiGe alloys have been employed. ${ }^{2}$ The magnitude of the band discontinuities between these alloys and $\mathrm{Si}$ is limited by the lattice mismatch and relatively small difference in band gaps.

Epitaxy of other metal silicides on Si has been reported, including $\mathrm{CoSi}_{2},{ }^{4} \mathrm{NiSi}_{2},{ }^{5} \mathrm{PdSi}_{2},{ }^{6} \mathrm{PtSi}^{6}{ }^{6} \mathrm{YSi}_{2-\mathrm{x}},{ }^{7}$ and numerous refractory metal silicides. ${ }^{8}$ High-quality epitaxial growth has only been demonstrated for materials with cubic structures though, and the best results have been obtained for $\mathrm{CoSi}_{2}$ and $\mathrm{NiSi}_{2}{ }^{4,5}$ Both of these materiais possess a close lattice match to $\mathrm{Si}$ and have the cubic-fluorite structure, which is very similar to the diamond structure of $\mathrm{Si}$.

Chromium disilicide is a semiconducting silicide with a band gap of $0.3 \mathrm{eV} .{ }^{9}$ It possesses a hexagonal (space group $\mathrm{P}_{2} 22$, or $\mathrm{C} 40$ ) structure with $a_{0}=0.4428 \mathrm{~nm}$ and $c_{0}=0.636 \mathrm{~nm}$, and lattice matches $\mathrm{Si}(111)$ to $0.1 \%$ [Fig 1(a)]. If $\mathrm{CrSi}_{2}$ layers with high-quality interfaces can be grown on $\mathrm{Si}$, heterojunction devices with large band discontinuities and small strain would be possible. In addition, the availability of a small-band-gap semiconductor on Si would allow the monolithic integration of $\mathrm{CrSi}_{2}$-based devices such as medium-wavelength infrared detectors with signal-processing circuitry based in the Si.

Localized epitaxy of $\mathrm{CrSi}_{2}$ on $\mathrm{Si}(111)$ has been reported by Shiau et $a l^{10}$ for layers formed by $\mathrm{Cr}$ deposition in a vacuum of $0.3-2.0 \times 10^{-6}$ Torr. The samples were annealed after deposition in a separate vacuum furnace, and epitaxial regions of $<2 \mu \mathrm{m}$ extent were obtained. In addition, Shiau $e t$ al. ${ }^{11}$ have reported localized epitaxy of $\mathrm{CrSi}_{2}$ on $\mathrm{Si}(100)$ and $\mathrm{Si}(110)$, though the quality of the layers was poorer than on the (111) orientation. We report here the first growth of $\mathrm{CrSi}_{2}$ on $\mathrm{Si}(111)$ in a commericial molecular-beam epitaxy (MBE) system, with a base pressure in the low $10^{-11}$ Torr range.

\section{EXPERIMENTAL PROCEDURE}

Si(111) wafers, 2-in. diameter, were chemically cleaned by a process which leaves a thin, protective oxide on the wafers. ${ }^{12}$ The wafers were then loaded in a RIBER EVA 32 molecular-beam epitaxy machine, and the oxide was removed by either a Si beam ${ }^{13}$ or by thermal desorption. ${ }^{12}$ This system is equipped with separate electron-beam evaporators which are used for Si and $\mathrm{Cr}$.

The $\mathrm{CrSi}_{2}$ layers were grown by four different techniques, which are described as follows. The first of these consists of the deposition of Cr on a room-temperature Si substrate followed by annealing at elevated temperature. While this technique has been referred to as solid-phase epitaxy in work on $\mathrm{CoSi}_{2}$ and $\mathrm{NiSi}_{2}$, we will refer to it as solid-phase reactive epitaxy (SRE) to signify the fact that considerable interdiffusion of the constituent species must occur. The second technique consists of the deposition of $\mathrm{Cr}$ on a hot $\mathrm{Si}$ substrate, and is denoted reactive deposition epitaxy (RDE), in keeping with earlier work on $\mathrm{CoSi}_{2}$. In the third technique, $\mathrm{Cr}$ and $\mathrm{Si}$ are codeposited at room temperature in stoichiometric ratio and annealed at elevated temperature, which we will refer to as solid-phase epitaxy (SPE). The final technique consists of codeposition of $\mathrm{Cr}$ and $\mathrm{Si}$ in stoichiometric ratio on a hot $\mathrm{Si}$ substrate, and is referred to as $\mathrm{MBE}$, also in keeping with earlier work on $\mathrm{CoSi}_{2}$. For all four techniques, the temperature reported is the maximum temperature to which the sample was subjected.

The wafers were monitored in situ during growth using reflection high-energy electron diffraction (RHEED). After removal from the MBE system, samples were analyzed by scanning electron microscopy (SEM), transmission electron microscopy (TEM), and Rutherford backscattering spectroscopy (RBS). 


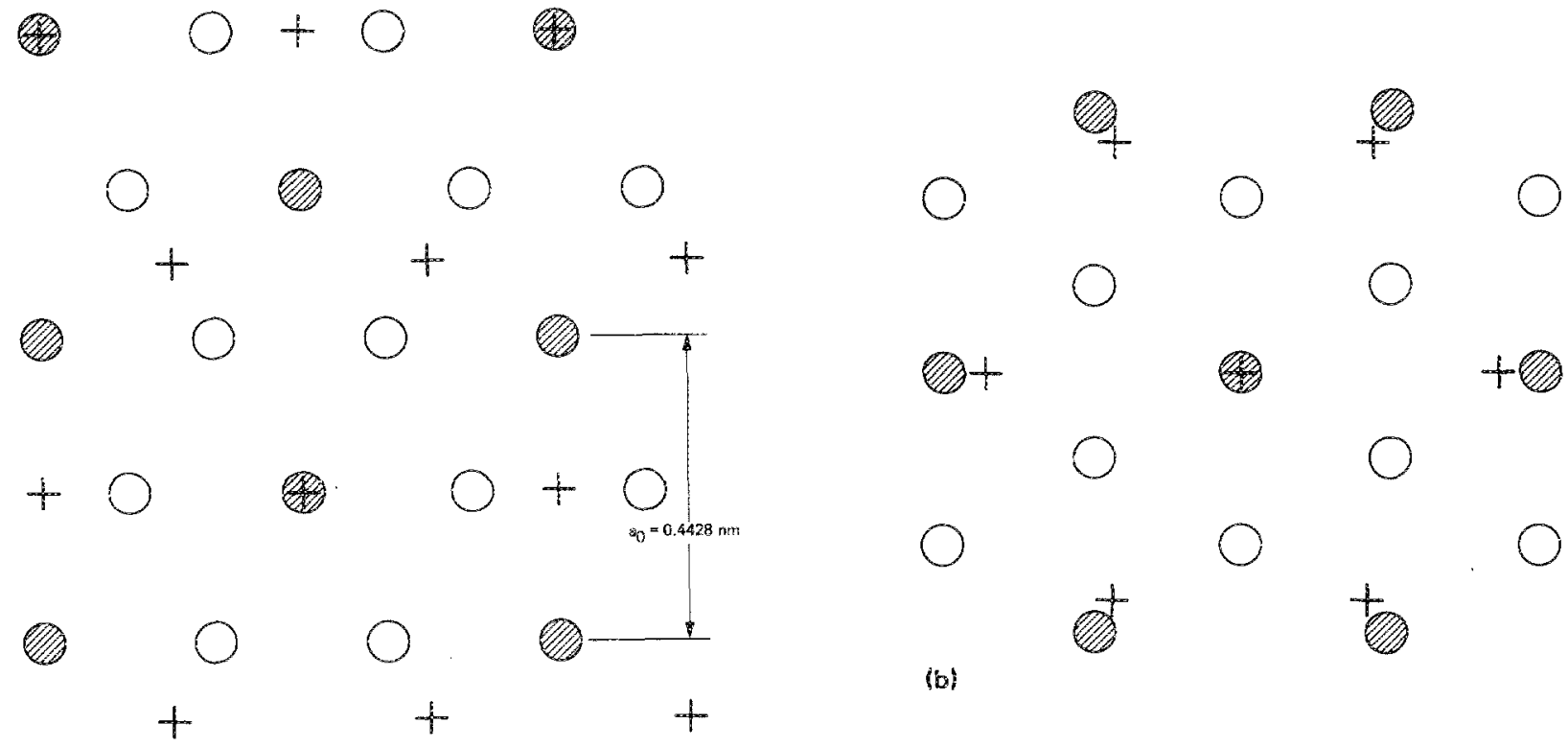

(a)

FIG. 1. Schematic diagrams of a single basal plane of the $\mathrm{CrSi}$, lattice superimposed on a single plane of the Si lattice, where represents $\mathrm{Cr}$ atoms, $\mathrm{O}$ represents $\mathrm{Si}$ atoms in $\mathrm{CrSi}_{2}$, and + represents $\mathrm{Si}$ atoms in the Si(111) substrate. (a) One possible alignment with $0.1 \%$ mismatch and (b) one possible alignment with $14 \%$ mismatch. In (b), the $\mathrm{CrSi}$ lattice is rotated by $30^{\circ}$ with respect to the $\$$ i lattice relative to (a).

\section{RESURTS}

Four different 10-nm-thick $\mathrm{CrSi}_{2}$ layers have been grown, three by SRE at 580,650 , and $720^{\circ} \mathrm{C}$, and one by RDE at $650^{\circ} \mathrm{C}$. SEM micrographs of these samples are shown in Fig. 2. An increasing tendency to form islands is noted in the SRE samples at higher temperatures, while the RDE sample exhibits smaller islands. RHEED patterns of the SRE layers show very diffuse streaks at $580^{\circ} \mathrm{C}$ and sharper streaks at higher temperatures (though the Si substrate may be contributing in this case). The RHEED pattern for the RDE layer shows spots indicative of transmission of the electron beam through islands. Planar TEM analysis of this sample reveals pronounced faceting of many of these islands. In addition, TEM analysis shows that all of these $\mathrm{CrSi}_{2}$ layers consist predominantly of grains with one of two orientations. In the first of these, the $\mathrm{CrSi}_{2}$ is oriented as expected (the orientation with a $0.1 \%$ mismatch) with respect to the $\mathrm{Si}$ substrate, i.e., with $\mathrm{CrSi}_{2}(0001) / / \mathrm{Si}(111)$ and $\mathrm{CrSi}_{2}[10 \overline{10}] / / \mathrm{Si}[101]$. In the second, the $\mathrm{CrSi}_{2}$ is rotated $30^{\circ}$ with respect to the $S i$ substrate about the surface normal relative to the first case, i.e., with $\mathrm{CrSi}_{2}(0001) / / \mathrm{Si}(111)$ and $\mathrm{CrSi}_{2}[1120] / / \mathrm{Si}[10 \mathrm{1}]$. The mismatch for this rotated orientation is $14 \%$. Electron diffraction patterns taken from

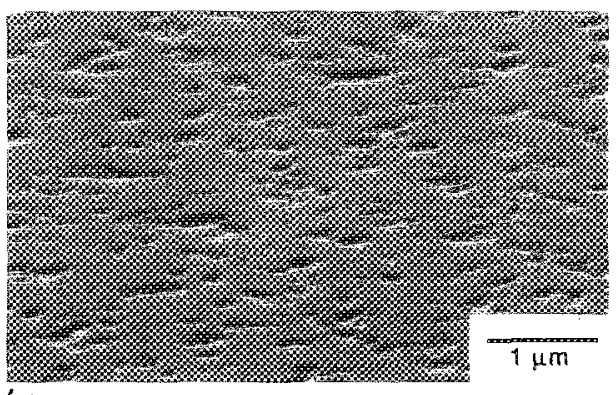

(a)

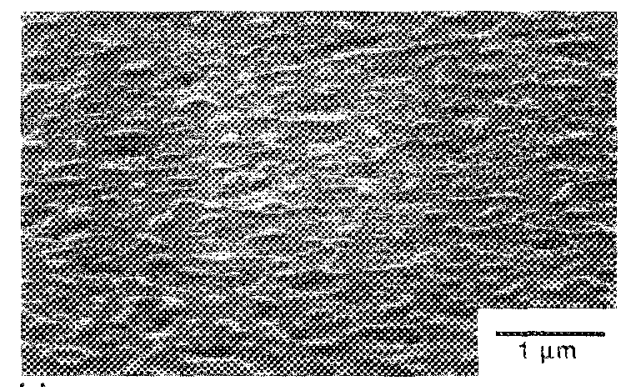

(c)

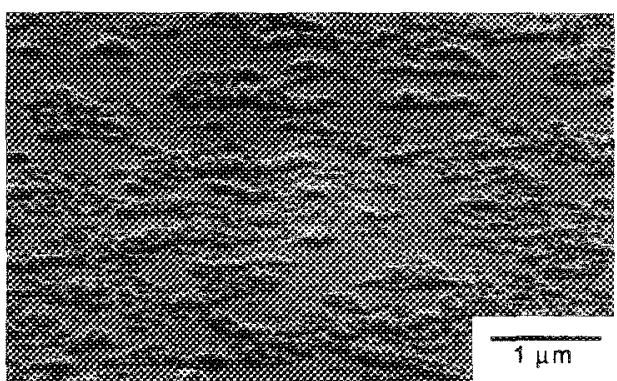

(b)

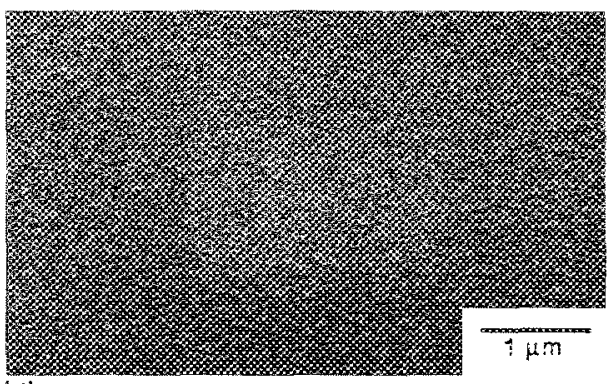

(d)
FIG. 2. SEM micrographs of 10 nm-thick $\mathrm{CrSi}_{2}$ layers grown on Si(111) using SRE with annealing at (a) 580 , (b) 650 , and (c) $720^{\circ} \mathrm{C}$, and (d) using $R D E$ at $650^{\circ} \mathrm{C}$. The microscope stage was tilted $70^{\circ}$ with respect to the electron beam for these layers. 

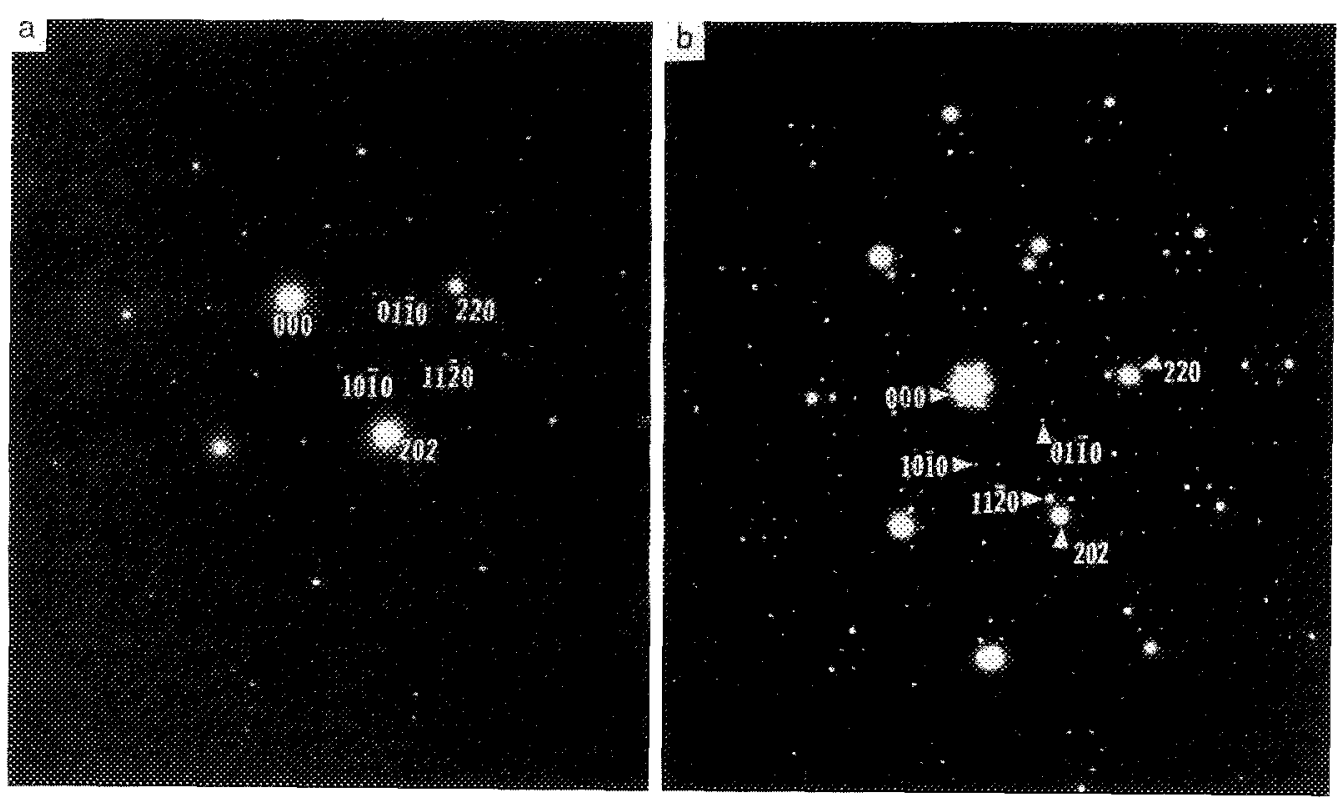

FIG. 3. Transmission electron diffraction (TED) patterns taken from a 20-nm-thick $\mathrm{CrSi}_{2}$ layer grown by SRE at $720^{\circ} \mathrm{C}$. (a) A TED pattern from an area exhibiting the expected epitaxial relationship [Fig. 1(a)], namely, $\quad \mathrm{CrSi}_{2}((0) 1) / / \mathrm{Si}(111)$ with $\mathrm{CrSi}_{2}[10 \overline{10} / / \mathrm{Si}[10 \overline{1}]$. (b) A TED pattern from an area exhibiting a $30^{\circ}$ rotation about the Si surface normal [Fig. 1(a)], $\mathrm{CrSi}_{2}(0001) / /$ $\mathrm{Si}(111)$ with $\mathrm{CrSi}_{2}[11 \overline{2} 0] / /$ $\mathrm{Si}[10 \overline{1}]$.

regions of planar samples with these two orientations are shown in Fig. 3. [Note that the extra spots in Fig. 3(b) are due to double diffaction.] Bright- and dark-field TEM micrographs taken in cross section from the $720^{\circ} \mathrm{C}$ SRE sample are shown in Fig. 4, which shows a region of the sample having an island with each of these orientations.

Thicker layers $(210 \mathrm{~nm}$ ) have also been examined, and in this case all four growth techniques discussed above were employed. Figure 5 shows SEM micrographs of $\mathrm{CrSi}_{2}$ layers grown with these four techniques, where the maximum temperature is $650^{\circ} \mathrm{C}$ for all layers. RHEED patterns for these samples show a streak pattern for the MBE layer, a more diffuse streak pattern for the RDE layer, and almost featureless patterns for the SRE and SPE layers. Very faint rings and spots are also seen in the RHEED patiern for the MHE layer, indicating the presense of some misoriented grains. Analysis of the RDE, SPE, and MBE layers with RBS shows them to be stoichiometric $\mathrm{CrSi}_{2}$ throughout the depth of the layer, and there is no evidence of tailing of $\mathrm{Cr}$ into the substrate. However, no channeling was observed, which suggests that there is no single epitaxial orientation occurring over a large fraction of the layer. On the other hand, the streak pattern observed for the MBE layer by RHEED suggests that much of the layer has a single orientation. Preliminary TEM results show some grains with the expected orientational relationship, but additional grains of other orientations.

While the surface morphology of the SPE sample is much better than that of the other samples, the RHEED pattern suggests that this layer did not fully recrystallize. As the RHEED pattern of the MBE layer exhibited the sharpest streaks, and SEM revealed the largest single-crystal grains for this sample, higher temperature MBE growth was examined. The SEM micrographs of Fig. 6 show MBE layers grown at 650,750 , and $825^{\circ} \mathrm{C}$. The grain size is seen to increase significantly with temperature, but RBS analysis of the $825^{\circ} \mathrm{C}$ sample still failed to reveal channeling.

\section{DISCUSSION}

The initial attempts reported herein at growing $\mathrm{CrSi}_{2}$ epitaxial layers in an MBE machine have not been successful in producing single-crystal layers. This is due at least in part to

a $1000 \AA$

(a)

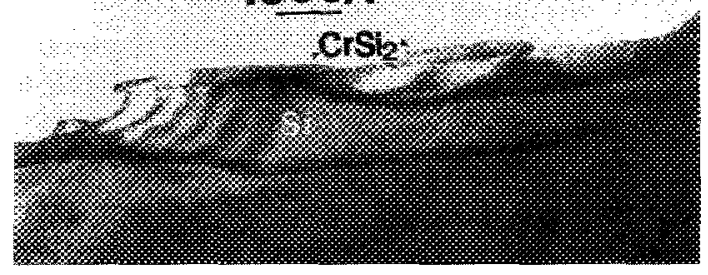

(b)

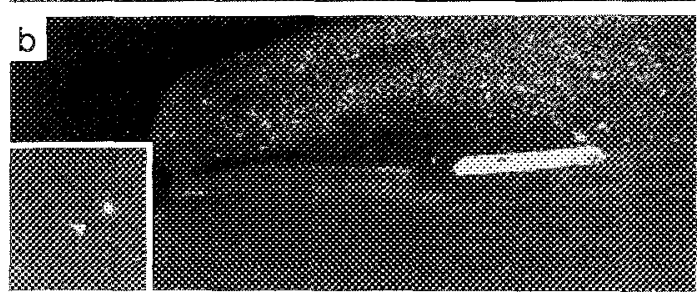

(c)

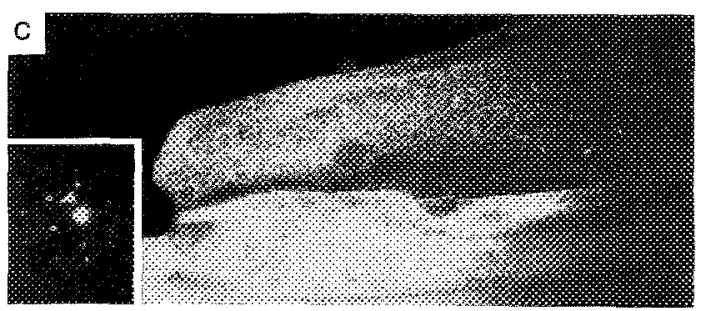

FIG. 4. TEM micrographs of a 10-nm-thick $\mathrm{CrSi}_{2}$ layer grown on $\mathrm{Si}(111)$ using the SRE technique with an annealing temperature of $720^{\circ} \mathrm{C}$. (a) A bright-ficld micrograph showing two $\mathrm{CrSi}$, islands (marked by arrows) on the Si substrate. (b) A dark-field micrograph formed with a diffraction spot (see inset TED pattern) from the unrotated material, showing that the island on the right-hand side is of this orientation. (c) A dark-field micrograph formed with a diffraction spot (see inset TED pattern) from the $30^{\circ}$ rotated material, showing that the island on the left-hand side is of this orientation. (All three micrographs have the same scale, and the layer on top of the $\mathrm{CrSi}_{2}$ is epoxy.) 


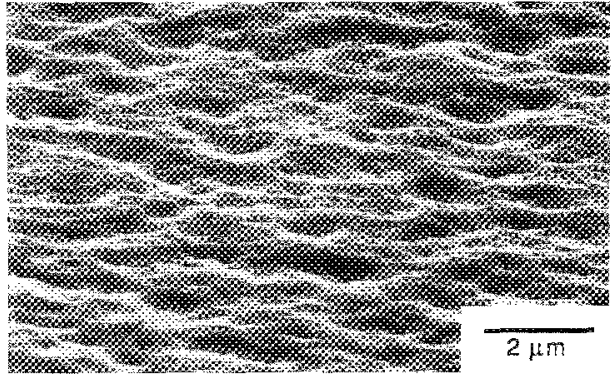

(a)

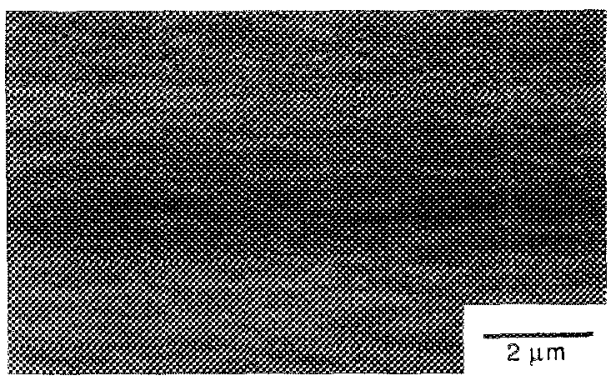

(e)

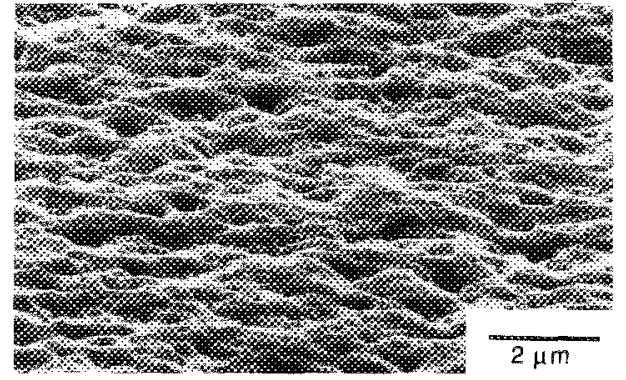

(b)

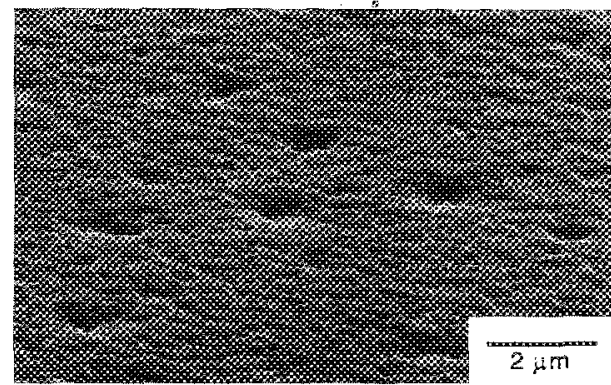

(d)
FIG. 5. SEM micrographs of 210 tmithick CrSi layers grown on Si(111) using (a) SRE, (b) RDE, (c) SPE, and (d) MBE growth techniques. The maximum temperature in all four cases is $650^{\circ} \mathrm{C}$, and the microscope stage was tilted $70^{\circ}$ with respect to the electron beam for these images.

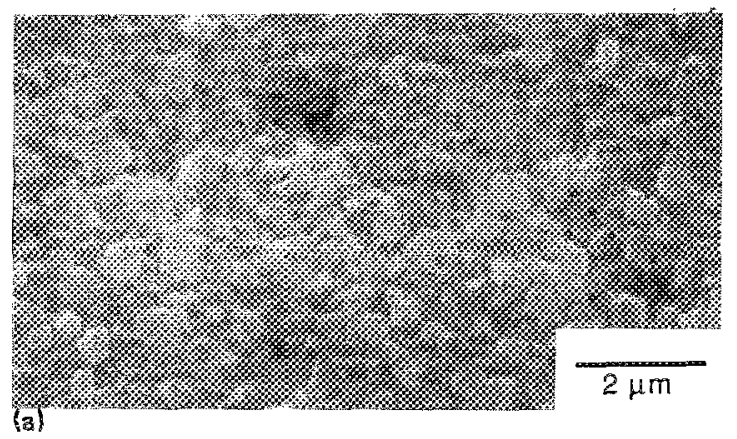

(3)

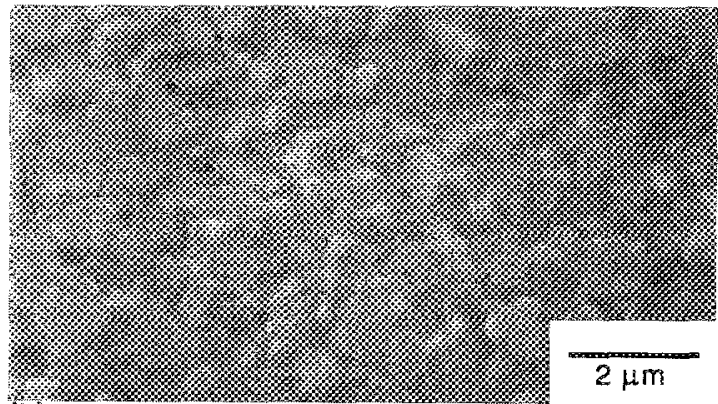

(b)

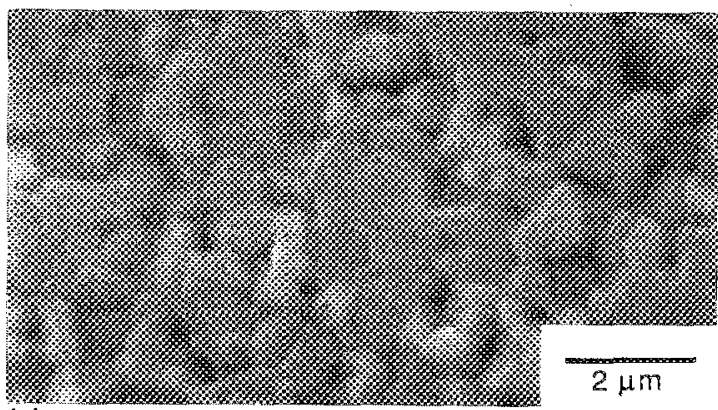

(c)

FrG. 6. SEM micrographs of 210 -nm-thick CrSi layers grown on $\mathrm{Si}(111)$ using the MBE growith technique at (a) 650 , (b) 750 , and (c) $825^{\circ} \mathrm{C}$. In contrast to the micrographs of Figs. 2 and 5 , the siage is not tilted in this case. the tendency of $\mathrm{CrSi}_{2}$ layers on $\mathrm{Si}(111)$ to form islands which assume more than one orientation with respect to the Si substrate. In addition to the expected epitaxial relationship, a $30^{\circ}$-rotated relationship is commonly observed. While this orientation possesses a high degree of symmetry with respect to the Si substrate, the mismatch is $14 \%$ (as compared to $0.1 \%$ in the unrotated case). As seen in Fig. 1 (a), the epitaxial relationship in the $0.1 \%$-mismatch case has a relatively large unit cell, so that a variety of bonding arrangements must occur. In contrast, were it not for the large mismatch in the rotated case, a one-to-one correlation of Cr atoms and substrate $\mathrm{Si}$ atoms would be found. Epitaxial nucleation of the rotation orientation does not therefore seem unikely, but the large mismatch should prevent grains of appreciable size from developing. The fact that they are observed may indicate weak interfacial bonding between $\mathrm{CrSi}_{2}$ and $\mathrm{Si}$. We note that this $30^{\circ}$-rotated orientation is not reported by Shiau et al. ${ }^{10}$ This may be due to the difference in cleanliness between the two studies.

It appears as if there is a greater variety of orientations present in the thicker layers than in the thin layers, though TEM analysis of these layers is incomplete at this time. The increasing grain size noted with increasing temperature for MBE layers implies that higher temperatures may aid in improving film quality. However, better understanding of the nucleation of islands is needed; in particular, a growth technique which results in the nucleation of islands predominantly of a single type is desired.

\section{SUMMARY AND GONCLUSIONS}

$\mathrm{CrSi}_{2}$ layers grown on Si(111) exhibit a strong tendency to form islands. Two particular epitaxial relationships are commonly observed, but other orientations are observed as well. The commonly occurring orientations are 
$\mathrm{CrSi}_{2}(0001) / / \mathrm{Si}(111)$ with $\mathrm{CrSi}_{2}[10 \overline{1} 0] / / \mathrm{Si}[10 \overline{1}]$ and $\mathrm{CrSi}_{2}(0001) / / \mathrm{Si}(111)$ with $\mathrm{CrSi}_{2}[11 \overline{20} 0] / / \mathrm{Si}[10 \overline{1}]$. The latter case represents a $30^{\circ}$ rotation of the $\mathrm{CrSi}_{2}$ layer about the surface normal as compared to the former case. For thick layers, the largest grains and best-quality RHEED pattern are obtained for layers grown by $\mathrm{MBE}$ (codeposition of $\mathrm{Cr}$ and $\mathrm{Si}$ at elevated temperature). Furthermore, the grain size is observed to increase with increasing substrate temperature, with grains of $1-2 \mu \mathrm{m}$ extent observed in a layer deposited at $825^{\circ} \mathrm{C}$.

\section{ACKNOWLEDGMENTS}

We would like to thank R. Ruiz for SEM analysis and $B$. Pate for technical assistance. The research described in this paper was carried out by the Jet Propulsion Laboratory (JPL), California Institute of Technology, and was supported by the Strategic Defense Initiative Organization, Innovative Science and Technology Office and the National Aeronautics and Space Administration. The work was performed as part of the JPL's Center for Space Microelectronics Technology. TEM work was performed at the University of Southern California, Center for Microscopy and Microanalysis, and was supported by a University of Southern Cali- fornia Faculty Research and Innovation Fund award to Dr. J. H. Mazur.

${ }^{1}$ A. Kastalsky, S. Luryi, J. C. Bean, and T. T. Sheng, in Proceedings of the First Iniernational Symposium on Si MBE, edited by J. C. Bean (Electrochemical Society, Pennington, NJ, 1985), pp. 406-411.

${ }^{2}$ G. Abstreiter, H. Brugger, T. Wolf, H. Jorke, and H. J. Herzog, Phys. Rev. I.ett. 54, 2441 (1985).

${ }^{3}$ R. Fischer, H. Morkoç, D. A. Neumann, H. Zabel, C. Choi, N. Otsuka, M. Longerbone, and L. P. Erickson, J. Appl. Phys. 60, 1640 (1986)

${ }^{4}$ R. T. Tung, J. C. Bean, J. M. Gibson, J. M. Poate, and D. C. Jacobson, Appl. Phys. Lett. 40, 684 (1982).

${ }^{5}$ R. T. Tung, J. M. Gibson, and J. M. Poate, Appl. Phys. Lett. 42, 888 (1983).

${ }^{6} \mathrm{H}$. Ishiwara, K. Hikosaka, M. Nagatoma, and S. Furukawa, Surf. Sci. 86, 711 (1979).

${ }^{7}$ M. Gurvitch, A. F. J. Levi, R. T. Tung, and S. Nakahara, Appl. Phys. Lett. 51, 311 (1987),

${ }^{8}$ L. J. Chen, H. C. Cheng, and W. T. Lin, in Materials Research Society Symposia Proceedings, edited by R. J. Nemanich, P. S. Ho, and S. S. Lau (Materials Research Society, Pittsburgh, PA, 1986), Vol. 54, pp. 245254.

${ }^{9}$ F. Nava, T. Tien, and K. N. Tu, J. Appl. Phys. 57, 2018 (1985).

${ }^{10}$ F. Y. Shiau, H. C. Cheng, and L. J. Chen, Appl. Phys. Lett. 45, 524 (1984).

'F. Y. Shiau, H. C. Cheng, and L. J. Chen, J. Appl. Phys. 59, 2784 (1986).

${ }^{12}$ A. Ishizaka and Y. Shiraki, J. Electrochem. Soc. 133, 666 (1987).

${ }^{13}$ K. Kugiyama, Y. Hirofuji, and N. Matsuo, Jpn. J. Appl. Phys. 24, 564 (1985). 